\title{
Risk Factors, Diagnosis, and Management of Halitosis in Children: A Comprehensive Review
}

\author{
Omar A Bawazir
}

\begin{abstract}
Aim: The present manuscript aims at providing a comprehensive overview of the epidemiology, risk factors, diagnostic aids, and management of halitosis in a pediatric population.

Background: Halitosis refers to bad breath from the mouth that seems unpleasant or offensive to others. This condition is prevalent worldwide, including all age-groups. However, studies on the pediatric population are limited. Self-confidence and personality development are important factors that the child builds up during their developmental stages. Halitosis may affect them both on personal and social aspects; hence, it is important to have beforehand knowledge and the modes to intercept it, paving the way to a positive growth of the child.

Review results: On reviewing the literature, the common risk factors for halitosis in children constituted predominantly oral factors that varied from poor oral hygiene, gingivitis, periodontal diseases, dental caries, tongue coating, mouth breathing, and so on. Their diagnosis commonly included the organoleptic test, sulfide monitoring, and gas chromatography along with the assessment of questionnaire by the parents and older children. The management was predominantly directed toward the identified source of halitosis along with instructions and counseling on the benefits of a good oral hygiene regime.

Conclusion: The importance of cultivating a day-to-day practice of oral hygiene regime in the children will not only accustom them toward the benefits of good oral health but also help them to tackle halitosis and in turn their inhibitions associated with it.

Clinical significance: Halitosis, though a prevalent condition experienced at various stages of life, is crucial to identify and manage, especially in the pediatric population owing to its influence on personal and on social aspects of life.

Keywords: Children, Halitosis, Organoleptic test, Oral malodor, Pediatric population, Volatile sulfur compounds.

The Journal of Contemporary Dental Practice (2021): 10.5005/jp-journals-10024-3139
\end{abstract}

\section{INTRODUCTION}

Halitosis originates from the Latin word "halitus," which means breath, and the Greek suffix osis refers to a condition or pathologic process. ${ }^{1}$ This prevalent disorder, also referred to as bad breath, oral malodor, or fetor oris, is used to explain the unpleasant breath emitted from the mouth of either oral or nonoral origin that seems unpleasant or offensive to others. ${ }^{2,3}$ Its wide prevalence among different age-groups necessitates a thorough knowledge of this entity, especially the pediatric population owing to the limited documentation of literature when compared to the adults. Evidence suggests that halitosis in children is related primarily to oral factors as seen in adults. The prevalence of oral microbiological factors, poor oral hygiene, and nasal-associated factors, such as postnasal drip, Helicobacter pylori infection, parasitic infection, sinusitis, tonsillitis, allergies, dry mouth, and mouth-breathing habit, commonly encountered in children are some of the etiological factors considered to be responsible for halitosis in them. ${ }^{4}$ The identification of oral halitosis in younger children may be limited unless attention is paid and recognized by the caregivers. Further, the influence of halitosis on the social and personal aspects of life, self-confidence, self-esteem, and personality buildup, which are crucial in the developing age of the child could be disturbed. ${ }^{5}$ Therefore, the identification and management of this entity in children are crucial, wherein voluntary assessment may be doubtful unless evaluated. Hence, this comprehensive review aims at assimilating its epidemiology, etiopathogenesis, diagnostic aids, and management options directed toward the pediatric population.
Department of Pediatric Dentistry and Orthodontics, College of Dentistry, King Saud University, Riyadh, Saudi Arabia

Corresponding Author: Omar A Bawazir, Department of Pediatric Dentistry and Orthodontics, College of Dentistry, King Saud University, Riyadh, Saudi Arabia, e-mail: obawazir@ksu.edu.sa

How to cite this article: Bawazir OA. Risk Factors, Diagnosis, and Management of Halitosis in Children: A Comprehensive Review. J Contemp Dent Pract 2021;22(8):959-963.

Source of support: Nil

Conflict of interest: None

\section{Pathophysiology}

Halitosis arises from the production of volatile compounds mainly sulfur compounds (VSCs), aromatic, nitrogen-containing compounds, alcohols, ketones, and others. These compounds are released due to pathological or nonpathological process and from either oral or extraoral origin. ${ }^{6}$ The whole process is initiated by the breakdown of components of the epithelial cells, salivary or serum proteins, and food debris that could be present on the dorsum of the tongue, in the saliva, or in the periodontal pockets by the action of a wide range of oral anaerobic microorganisms, which degrade organic substances and produce odorous compounds. ${ }^{7,8}$ Hundreds of bacteria have been identified in the oral cavity that are capable of producing VSCs; however, a wide diversity is found in the literature regarding halitosis-causing bacteria, which suggests complex interactions between those bacterial species particularly the

(c) The Author(s). 2021 Open Access This article is distributed under the terms of the Creative Commons Attribution 4.0 International License (https://creativecommons. org/licenses/by-nc/4.0/), which permits unrestricted use, distribution, and non-commercial reproduction in any medium, provided you give appropriate credit to the original author(s) and the source, provide a link to the Creative Commons license, and indicate if changes were made. The Creative Commons Public Domain Dedication waiver (http://creativecommons.org/publicdomain/zero/1.0/) applies to the data made available in this article, unless otherwise stated. 
gram-negative anaerobes and proteolytic obligate anaerobes..$^{2,8,9}$ VSCs, particularly hydrogen sulfide $\left(\mathrm{H}_{2} \mathrm{~S}\right)$, methyl mercaptan $\left(\mathrm{CH}_{3} \mathrm{SH}\right)$, and dimethyl sulfide $\left(\mathrm{CH}_{3} \mathrm{SCH}_{3}\right)$, are considered the major components associated with halitosis. ${ }^{7,8,10}$ Both $\mathrm{H}_{2} \mathrm{~S}$ and $\mathrm{CH}_{3} \mathrm{SH}$ are considered the main contributors to oral halitosis, while $\mathrm{CH}_{3} \mathrm{SCH}_{3}$ was reported to be mainly contributing to extraoral and bloodborne halitosis, but may also contribute to oral halitosis., ${ }^{6,9,11}$

\section{Classification of Halitosis}

According to the International Society for Breath Odor Research, halitosis is classified into three main categories: genuine halitosis, pseudohalitosis, and halitophobia based on treatment needs. ${ }^{12}$ Genuine halitosis is the condition where breath malodor can be verified objectively and with an intensity beyond socially acceptable levels. It is further subdivided into physiologic halitosis with no readily apparent disease and pathologic halitosis, which is the result of an infective process with either an oral or extraoral origin. Pseudohalitosis is a condition wherein obvious malodor though not perceived, yet the patient insists on its presence. Lastly, halitophobia is the belief of the existence of malodor by a patient even after successful treatment of either genuine or pseudohalitosis. ${ }^{12}$

Being an apparently common phenomenon, it is governed by intrinsic (oral and extraoral) and extrinsic etiological factors, ${ }^{11,13}$ wherein oral causes account for approximately $90 \%$ of halitosis cases, ${ }^{8-10}$ while the extraoral causes to $10 \%$ of the reported cases, which may be a reflection of underlying systemic pathologies too at times. ${ }^{7,10,14}$

Halitosis is also classified as primary halitosis wherein reference to respiration exhaled by the lungs is considered to be the causative factor and secondary wherein the etiology is considered to be oral or upper airways. ${ }^{15}$

\section{Halitosis in Children}

\section{Prevalence of Halitosis in Children}

The prevalence of halitosis among the pediatric population has been investigated worldwide with varying estimations, between $5 \%$ and $76.3 \%,{ }^{5}$ attributed to differences in factors, such as sample size, diagnostic methods, and age-groups considered. ${ }^{16-20} \mathrm{~A}$ study in Italy by Villa et al. reported a prevalence of $37.6 \%$ of halitosis among 101 children of 6 to 16 years of age. ${ }^{16}$ Patil et al. conducted a study on 900 Indian children of 7 to 15 years of age and found a prevalence of halitosis in $40.9 \%$ with a significant association between age, oral hygiene status, and mouth breathing. ${ }^{17}$ While Kanehira et al reported $8 \%$ of 119 Japanese children with halitosis wherein mouth breathing was a major contributing factor; ${ }^{19}$ on the contrary, Ueno et al. reported $44.9 \%$ with halitosis in 768 Japanese children of 6 to 15 years of age, wherein a significant score of tongue coating was considered a contributing factor for halitosis. ${ }^{20} \mathrm{Nalçaci}$ et al. reported a prevalence of $14.5 \%$ in 628 Turkish children of 7 to 11 years with halitosis, significantly associated with the severity of dental caries. ${ }^{21}$ Therefore, irrespective of the contributing or associated factors that differed for halitosis among the geographies, its dominant prevalence among the pediatric population was undeniable.

\section{Associated Risk Factors for Halitosis in Children}

Numerous studies have evaluated and reported statistically the significant presence of certain oral and extraoral factors with halitosis, which is routinely encountered in the pediatric population.
The literature has documented the prevalence of mouth breathing habit among the pediatric population (50-56\%) ${ }^{22}$ and has analyzed the relationship between mouth breathing and halitosis. Mouthbreathing habit as a primary entity leads to the changes in salivary flow patterns and water imbalance, resulting in the surface dryness of the oral mucosa, thereby contributing to halitosis. Further, conditions such as dental caries, periodontal diseases may have a positive association with mouth breathing; chronic allergies, nasal, and throat infections may be associated with mouth breathing, all of which may directly or indirectly contribute to halitosis in their own respective ways. ${ }^{23}$ Studies by Motta et al., ${ }^{15}$ Kanehira et al., ${ }^{19}$ and Alqutami et al. ${ }^{24}$ have reported a significant increase in halitosis among mouth breathers.

Other significant oral contributing factors, such as poor oral hygiene, periodontal diseases, ${ }^{25-28}$ tongue coating, ${ }^{17,20,26-29}$ dental caries, and proximal restorations, ${ }^{25,30,31}$ pose as a risk factor in the development of halitosis in the pediatric population. Fissured tongue presents as a high risk of bacterial and food lodgment within the fissures and, therefore, enhances the tongue coating. ${ }^{32}$ Further, Ueno et al. stated in their study that children with 2 or 3 score of tongue-coating thickness were 3.28-fold times more prone to present with halitosis than with score 0 and 1 thickness of tongue coating, which can be considered as a significant risk factor. ${ }^{20}$

Though studies have reported a positive association between orthodontic appliances and halitosis, ${ }^{33,34}$ however, contradicting to former results, Keris et al. reported a negative association of fixed and removable space maintainers with halitosis. ${ }^{35}$

Dinc et al. investigated the presence of halitosis in 40 children of 5 to 15 years of age diagnosed with adenoid hypertrophy and whether adenoidectomy decreased it. They concluded that there was a significant association between them and treatment did present with an improvement of halitosis. They further suggested that in children with no other oral pathologic etiology for halitosis, adenoid hypertrophy needs to be evaluated. ${ }^{3}$ Therefore, this helps us to understand the multifactorial origin and association of halitosis with the risk factors evaluated.

\section{Diagnosis of Halitosis in Children}

Halitosis assessment methods are crucial as they help to detect its presence, classify the type of halitosis, its severity, and thereby determine the treatment protocol to be followed accordingly. ${ }^{6}$ These diagnostic aids range from simple to complex, being subjective to objective, and used unsophisticated to sophisticated equipment. ${ }^{6,8,10}$ Common diagnostic aids, such as organoleptic measurement, sulfide monitoring, gas chromatography, BANA test, and chemical sensors and not so common methods, such as quantifying $\beta$-galactosidase activity, salivary incubation test, ammonia monitoring, and ninhydrin method are used in detecting halitosis presence, differentiate pseudohalitosis and halitophobia, and also assess the severity of halitosis. ${ }^{6}$ However, studies on the pediatric population have reported the routine use of organoleptic measurement, sulfide monitoring, and gas chromatography in halitosis assessment. 17,19,20,25,26,29,31 Another important factor to be noted was the questionnaire assessment on the presence of halitosis in children either by caregivers or selfevaluation, which was an additional factor evaluated commonly in some studies. ${ }^{5}$

Organoleptic assessment, a sensory test, ${ }^{13}$ is defined as a method that can measure the strength of target odors and expresses the value in terms of a point or number with reference 
to a predefined organoleptic scale. It is the oldest, simplest method where the quality of the mouth and/or nasal air will be assessed organoleptically by a calibrated odor judge, ${ }^{6,36}$ from a specific distance and scored on a scale from 0 to 5 points. Though simple test yet faced with low reproducibility, the requirement of trained breath assessor, disease transmission risk, and test specificity are a few of its limitations. ${ }^{9,11}$

A sulfide monitor, an objective test, analyzes the total sulfur content of the patients' mouth air. ${ }^{13}$ Halimeter (Interscan, Chatsworth, CA, USA), introduced in the early 1990s, is portable, chair-side equipment, where a reading less than $100 \mathrm{ppb}$ is normal while 100 ppb or more is considered as halitosis. Though it has a relatively cost-effective value, ${ }^{37}$ it has a high sensitivity for hydrogen sulfide, yet low sensitivity for methyl mercaptan; false-positive readings due to other volatile vapors, such as acetone, ethanol, and methanol, are its limitations. ${ }^{13,38,39}$

A gas chromatograph is a sophisticated device that analyzes exhaled breath and can detect a wide range of odorous compounds and is considered by many studies to be the method of choice for differentiating and quantifying major halitosis-associated VSCs. ${ }^{11}$ The analysis of VSCs by gas chromatography is considered to be the most reliable measurement for the diagnosis of intra- and extraoral halitosis by detecting even low concentrations of VSCs to distinguish the origins of halitosis. ${ }^{6,11}$ Conventional gas chromatography is a relatively expensive, massive, and large apparatus that requires trained and experienced technicians and specialists. ${ }^{40} \mathrm{~A}$ small, portable version of conventional gas chromatography known as OralChroma $^{\mathrm{TM}}$ (Oral Chroma ${ }^{\mathrm{TM}}$, Abimedical, Osaka, Japan) became available in $2003,{ }^{41}$ wherein on evaluation, the concentration of each VSCs appears on the screen and halitosis is considered present if one or more of the VSCs gases exceeded the following cognitive threshold: $(\mathrm{H} 2 \mathrm{~S})>112 \mathrm{ppb},\left(\mathrm{CH}_{3} \mathrm{SH}\right)>26 \mathrm{ppb}$, and $\left(\mathrm{CH}_{3} \mathrm{SCH}_{3}\right)$ $>8$ ppb. $^{34,42}$

Villa et al. assessed halitosis in 101 children with the portable sulfide monitor and Halimeter ${ }^{\circ} ;{ }^{16}$ Guedes et al. considered organoleptic test in his cross-sectional study on 150 children; ${ }^{43}$ Dinc et al. assessed halitosis in 40 children with adenoid vegetation using the portable gas chromatograph OralChroma; ${ }^{3}$ Keceli et al. determined halitosis in 151 children using both the organoleptic and sulfide monitoring; ${ }^{29}$ Motta et al. used the Breath Alert ${ }^{\mathrm{TM}}$ on 55 children to assess halitosis, ${ }^{15}$ and Ueno et al. considered organoleptic assessment method for detection of halitosis in 768 children ${ }^{20}$ and so on. Some studies have even evaluated the differences between the tests for halitosis and their significant relationship in the pediatric population. In a study by Petrini et al., 50 children were assessed for halitosis using organoleptic test and quantification of salivary $\beta$-galactosidases, wherein they reported that the salivary $\beta$-galactosidases level was significantly related to organoleptic scores along with other clinical evaluated parameters. ${ }^{44}$ Lin et al., compared organoleptic test and Halimeter on 30 children, wherein the results were inconsistent. The authors concluded that their results may suggest the involvement of other factors for halitosis in the pediatric population. ${ }^{25}$ These above-cited studies and more documented in the literature help us to understand the different methods used for the detection of halitosis in children.

\section{Management of Halitosis in Children}

Identification of the presence of halitosis, followed by the type and its severity allows for an effective management protocol. The presence of microorganisms in the oral cavity and their role in the etiology of halitosis are well-documented. Also, poor oral hygiene, tongue coating, dental caries, gingivitis, periodontitis, and periodontal abscess wherein the microorganisms' role is undeniable may contribute to halitosis. Therefore, the primary aim of the management in such a scenario would be mechanically reducing the load of the microbial biofilm by enforcing a good oral hygiene regime, such as routine toothbrushing, flossing, tongue cleaning, and mouth rinses. ${ }^{9,26-29,45}$ It is also advisable to inculcate the habit of swishing the mouth after eating in children at a young age as a regular practice that would reduce food lodgment, microbial load, and even halitosis caused due to dietary foods, such as onion, garlic, to name a few. At times, the basic protocol followed as above is mostly successful in reducing halitosis to a greater extent in the pediatric population.

Addressing underlying evident pathology for halitosis follows the next step. Nonsurgical periodontal therapy comprising scaling, restoration of decayed teeth, and periodontal and pharmacological treatment as advised based on the oral examination findings is recommended by the practitioners. This would also aid in the reduction of the oral microbial load responsible for halitosis. In children with mouth breathing, identification of the possible etiological factors such as adenoid hypertrophy would aid in addressing the related halitosis. ${ }^{3}$

The literature documented that most of the children, who were assessed for halitosis, were of genuine halitosis with predominant oral pathologic causes; however, the severity did vary among them. Amir et al. reported a reduction in halitosis after providing oral hygiene instructions to the 24 children recruited in their study. ${ }^{28}$ Similar results were recorded by Nalçaci and Sönmez, whereupon improving oral hygiene and controlling gingival diseases in 30 caries-active children, a reduction of halitosis was noted. ${ }^{21}$ Kara et al. reported that the oral hygiene instruction and periodontal scaling were successful in reducing halitosis in 150 Turkish children. ${ }^{27}$ Keceli et al. reported significant reductions in both the VSC levels and organoleptic scores after instructing tongue brushing and a good oral hygiene in 69 children. ${ }^{29}$ In a study by Yilmaz et al., the authors reported that $H$. pylori eradication was beneficial in the treatment of children with a positive $H$. pylori antigen test and with halitosis. ${ }^{46}$ Similarly, Hoshi et al. and Sayedi et al. reported metronidazole effectiveness in children halitosis. ${ }^{4}$ Recently, AIMadhi et al. investigated the pre- and posteffect of full mouth rehabilitation on 57 children under general anesthesia on halitosis and reported a significant reduction of VSCs level in more than $50 \%$ of the sample measured by OralChroma ${ }^{\mathrm{Tm}}{ }^{31,47}$ Lastly, the importance of providing instructions and counseling to maintain a good oral hygiene to the caregivers and the children cannot be emphasized more, as they are the basic steps in the treatment protocol to be followed in the management of halitosis.

\section{Conclusion}

Halitosis, a prevalent condition worldwide, is also known for its influence on the personality and self-confidence of an individual. For the overall holistic development of a child, conditions such as halitosis need to be checked at the earliest. Identification of the risk factors, efficient diagnosis, and effective treatment protocol are to be addressed in countering halitosis. Most of the studies have emphasized the importance of educating and counseling the caregivers and children on regular oral hygiene, which aids in its prevention and forms a part of the treatment too. 


\section{References}

1. Attia EL, Marshall KG. Halitosis. Can Med Assoc J 1982;126(11):1281. Available at: https://pubmed.ncbi.nlm.nih.gov/7074455/

2. Cortelli JR, Barbosa MD, Westphal MA. Halitosis: a review of associated factors and therapeutic approach. Braz Oral Res 2008;22:44-54. DOI: 10.1590/s1806-83242008000500007.

3. Dinc ME, Altundag A, Dizdar D, et al. An objective assessment of halitosis in children with adenoid vegetation during pre-and postoperative period. Int J Pediatr Otorhinolaryngol 2016;88:47-51. DOI: $10.1016 / j . j$.jporl.2016.06.042.

4. Sayedi SJ, Modaresi MR, Saneian H. Effect of metronidazole on halitosis of 2 to 10 years old children. Iran J Pediatr 2015;25(1):e252. DOI: 10.5812/ijp.252.

5. Aliyu I, Lawal TO. Perception and awareness of halitosis in children by caregivers seen in our pediatric outpatient department. SRM J Res Dent Sci 2018;9(2):63. DOI: 10.4103/srmjrds.srmjrds_81_17.

6. Aylıkcı BU, Çolak H. Halitosis: from diagnosis to management. J Nat Sci Biol Med 2013;4(1):14. DOI: 10.4103/0976-9668.107255.

7. Bollen CM, Beikler T. Halitosis: the multidisciplinary approach. Int J Oral Sci 2012;4:55-63. DOI: 10.1038/ijos.2012.39.

8. Hughes FJ, McNab R. Oral malodour - a review. Arch Oral Biol 2008;53(1):S8-S12. DOI: 10.1016/S0003-9969(08)70003-7.

9. van den Broek AMWT, Feenstra $L$, de Baat $C$. A review of the current literature on aetiology and measurement methods of halitosis. J Dent 2007;35:627-635. DOI: 10.1016/j.jdent.2007.04.009.

10. Porter SR, Scully C. Oral malodour (halitosis). Br Med J 2006;333(7569):632-635. DOI: 10.1136/bmj.38954.631968.AE.

11. Tangerman A, Winkel EG. Intra- and extra-oral halitosis: finding of a new form of extra-oral blood-borne halitosis caused by dimethyl sulphide. J Clin Periodontol 2007;34(9):748-755. DOI: 10.1111/j.1600051X.2007.01116.x.

12. Murata T, Yamaga $T$, lida $T$, et al. Classification and examination of halitosis. Int Dent J 2002;52:181-186. DOI: 10.1002/j.1875-595x.2002. tb00921.x.

13. Yaegaki K, Coil JM. Examination, classification, and treatment of halitosis; clinical perspectives. J Can Dent Assoc 2000;66(5):257-261. Available at: https://pubmed.ncbi.nlm.nih.gov/10833869/

14. Madhushankari GS, Yamunadevi A, Selvamani M, et al. Halitosis-an overview: part-I-classification, etiology, and pathophysiology of halitosis. J Pharm Bioallied Sci 2015;7(Suppl 2):S339. DOI: 10.4103/0975-7406.163441.

15. Motta LJ, Bachiega JC, Guedes CC, et al. Association between halitosis and mouth breathing in children. Clinics 2011;66(6):939-942. DOI: 10.1590/s1807-59322011000600003.

16. Villa A, Zollanvari A, Alterovitz $G$, et al. Prevalence of halitosis in children considering oral hygiene, gender and age. Int J Dent Hyg 2014;12(3):208-212. DOI: 10.1111/idh.12077.

17. Patil PS, Pujar P, Poornima S, et al. Prevalence of oral malodour and its relationship with oral parameters in Indian children aged 7-15 years. Eur Arch Paediatr Dent 2014;15(4):251-258. DOI: 10.1007/s40368-0140109-0.

18. Yoshida M, Takamori K, Ono $\mathrm{Y}$, et al. Study on halitosis children: questionnaire and halitosis test. Pediatr Dent J 2001;39:694-703. Available at: https://www.scopus.com/record/display.uri?eid=2-s2.04043113016\&origin=inward\&txGid=e18fb9bb8ce8aa6 dc3cc084251 0c59da\#

19. Kanehira T, Takehara J, Takahashi D, et al. Prevalence of oral malodor and the relationship with habitual mouth breathing in children. J Clin Pediatr Dent 2004;28(4):285-288. DOI: 10.17796/ jcpd.28.4.xp213r6534322m58.

20. Ueno $M$, Ohnuki $M$, Zaitsu $T$, et al. Prevalence and risk factors of halitosis in Japanese school children. Pediatr Int 2018;60(6):588-592. DOI: 10.1111/ped.13561.

21. Nalçacı R, Dülgergil T, Oba AA, et al. Prevalence of breath malodour in 7-11 year old children living in Middle Anatolia, Turkey. Community Dent Health 2008;(25):173-177. Available at: https://pubmed.ncbi. nlm.nih.gov/18839725/
22. Yamaguchi H, Tada S, Nakanishi Y, et al. Association between mouth breathing and atopic dermatitis in Japanese children 2-6 years old: a population-based cross-sectional study. PLoS One 2015;10(4):e0125916. DOI: 10.1371/journal.pone.0125916.

23. Triana BE, Ali AH, León IG. Mouth breathing and its relationship to some oral and medical conditions: physiopathological mechanisms involved. Rev Habanera Cien Méd 2016;15(2):200-212. Available at: http://scielo.sld.cu/pdf/rhcm/v15n2/rhcm08215.pdf

24. Alqutami J, Elger W, Grafe N, et al. Dental health, halitosis and mouth breathing in 10-to-15 year old children: a potential connection. Eur J Paediatr Dent 2019;20(4):274-279. DOI: 10.23804/ejpd.2019.20.04.03.

25. Lin MIH, Flaitz CM, Moretti AJ, et al. Evaluation of halitosis in children and mothers. Pediatr Dent 2003;25(6):553-558. Available at: https:// pubmed.ncbi.nlm.nih.gov/14733469/

26. Nalçaci R, Sönmez IS. Evaluation of oral malodor in children. Oral Surg Oral Med Oral Pathol Oral Radiol Endodontol 2008;106(3):384-388. DOI: 10.1016/j.tripleo.2008.03.001.

27. Kara C, Tezel A, Orbak R. Effect of oral hygiene instruction and scaling on oral malodour in a population of Turkish children with gingival inflammation. Int J Paediatr Dent 2006;16(6):399-404. DOI: 10.1111/j.1365-263X.2006.00769.x.

28. Amir E, Shimonov R, Rosenberg M. Halitosis in children. J Pediatr 1999;134(3):338-343. DOI: 10.1016/s0022-3476(99)70460-9.

29. Keceli T, Gulmez D, Dolgun A, et al. The relationship between tongue brushing and halitosis in children: a randomized controlled trial. Oral Dis 2015;21(1):66-73. DOI: 10.1111/odi.12210.

30. Gholami F, Minah GE, Turng BF. Oral malodor in children and volatile sulfur compound-producing bacteria in saliva: preliminary microbiological investigation. Pediatr Dent 1999;21(6):320-324. Available at: https://pubmed.ncbi.nlm.nih.gov/10509331/

31. Al Madhi NA, Sulimany AM, Alzoman HA, et al. Halitosis and associated risk factors in children: a cross-sectional study. J Contemp Dent Pract 2021;22(1):51-55. Available at: https://pubmed.ncbi.nlm.nih. gov/34002709/

32. Ziaei $\mathrm{N}$, Hosseinpour $\mathrm{S}$, Nazari $\mathrm{H}$, et al. Halitosis and its associated factors among Kermanshah high school students (2015). Clin Cosmet Investig Dent 2019;11:327. DOI: 10.2147/CCIDE.S215869.

33. Babacan H, Sokucu O, Marakoglu I, et al. Effect of fixed appliances on oral malodor. Am J Orthod Dentofac Orthop 2011;139(3):351-355. DOI: 10.1016/j.ajodo.2009.03.055.

34. Costacurta M, Petrini M, Biferi V, et al. The correlation between different techniques for the evaluation of oral malodour in children with and without orthodontic treatment. Eur J Paediatr Dent 2019;20(3):233-236. DOI: 10.23804/ejpd.2019.20.03.12.

35. Keris E, Atabek D, Güngör K. Effects of fixed and removable space maintainers on halitosis. BMC Oral Health 2016;16(1):99. Available at: https://bmcoralhealth.biomedcentral.com/articles/10.1186/s12903016-0297-6

36. Greenman J, Lenton P, Seemann R, et al. Organoleptic assessment of halitosis for dental professionals-general recommendations. J Breath Res 2014;8(1):017102. DOI: 10.1088/1752-7155/8/1/017102.

37. Romano F, Pigella E, Guzzi N, et al. Patients' self-assessment of oral malodour and its relationship with organoleptic scores and oral conditions. Int J Dent Hyg 2010;8(1):41-46. DOI: 10.1111/j.16015037.2009.00368.x.

38. Lee PPC, Mak WY, Newsome P. The aetiology and treatment of oral halitosis: an update. Hong Kong Med J 2004;10(6):414-418. Available at: https://pubmed.ncbi.nlm.nih.gov/15591601/

39. Patil SH, Kulloli A, Kella M. Unmasking oral malodor: a review. People's J Sci Res 2011;5(1):61-67. Available at: https://www.pjsr.org/ Jan12_pdf/12.\%20Suvarna\%20H\%2OPatil.pdf

40. Murata T, Rahardjo A, Fujiyama Y, et al. Development of a compact and simple gas chromatography for oral malodor measurement. J Periodontol 2006;77(7):1142-1147. DOI: 10.1902/jop.2006.050388.

41. Hanada M, Koda $\mathrm{H}$, Onaga $\mathrm{K}$, et al. Portable oral malodor analyzer using highly sensitive In2O3 gas sensor combined with a simple gas chromatography system. Anal Chim Acta 2003;475(1-2):27-35. DOI: 10.1016/S0003-2670(02)01038-3. 
42. Tangerman A, Winkel EG. The portable gas chromatograph OralChroma ${ }^{\mathrm{TM}}$ : a method of choice to detect oral and extraoral halitosis. J Breath Res 2008;2(1):017010. DOI: 10.1088/17527155/2/1/017010.

43. Guedes CC, Bussadori SK, Weber R, et al. Halitosis: prevalence and association with oral etiological factors in children and adolescents. J Breath Res 2019;13(2):026002. DOI: 10.1088/1752-7163/aafc6f.

44. Petrini $M$, Costacurta $M$, Ferrante $M$, et al. Association between the organoleptic scores, oral condition and salivary $\beta$-galactosidases in children affected by halitosis. Int J Dent Hyg 2014;12(3):213-218. DOI: 10.1111/idh.12083.
45. Van Den Broek AM, Feenstra $L$, et al. A review of the current literature on management of halitosis. Oral Dis 2008;14(1):30-39. DOI: 10.1111/j.1601-0825.2006.01350.x.

46. Yilmaz AE, Bilici M, Tonbul A, et al. Paediatric halitosis and Helicobacter pylori infection. J Coll Physicians Surg Pak 2012;22(1):27-30. Available at: https://pubmed.ncbi.nlm.nih.gov/22237186/

47. AlMadhi NA, Sulimany AM, Alzoman HA, Bawazir OA. Halitosis in Children Undergoing Full Mouth Rehabilitation under General Anesthesia. Children (Basel). 2021;8(2):149. DOI: 10.3390/children 8020149 . 